Supporting Information for

\title{
Color Toning of Mie Resonant Silicon Nanoparticle Color Inks
}

Takuma Okazaki,${ }^{\dagger}$ Hiroshi Sugimoto, ${ }^{*}, \dagger,+$ Tatsuki Hinamoto,${ }^{\dagger}$ Minoru Fujii ${ }^{\dagger}$

†Department of Electrical and Electronic Engineering, Graduate School of Engineering,

Kobe University, Rokkodai, Nada, Kobe 657-8501, Japan

¥JST-PRESTO, Honcho 4-1-8, Kawaguchi, Saitama 332-0012, Japan

*E-mail: sugimoto@eedept.kobe-u.ac.jp 

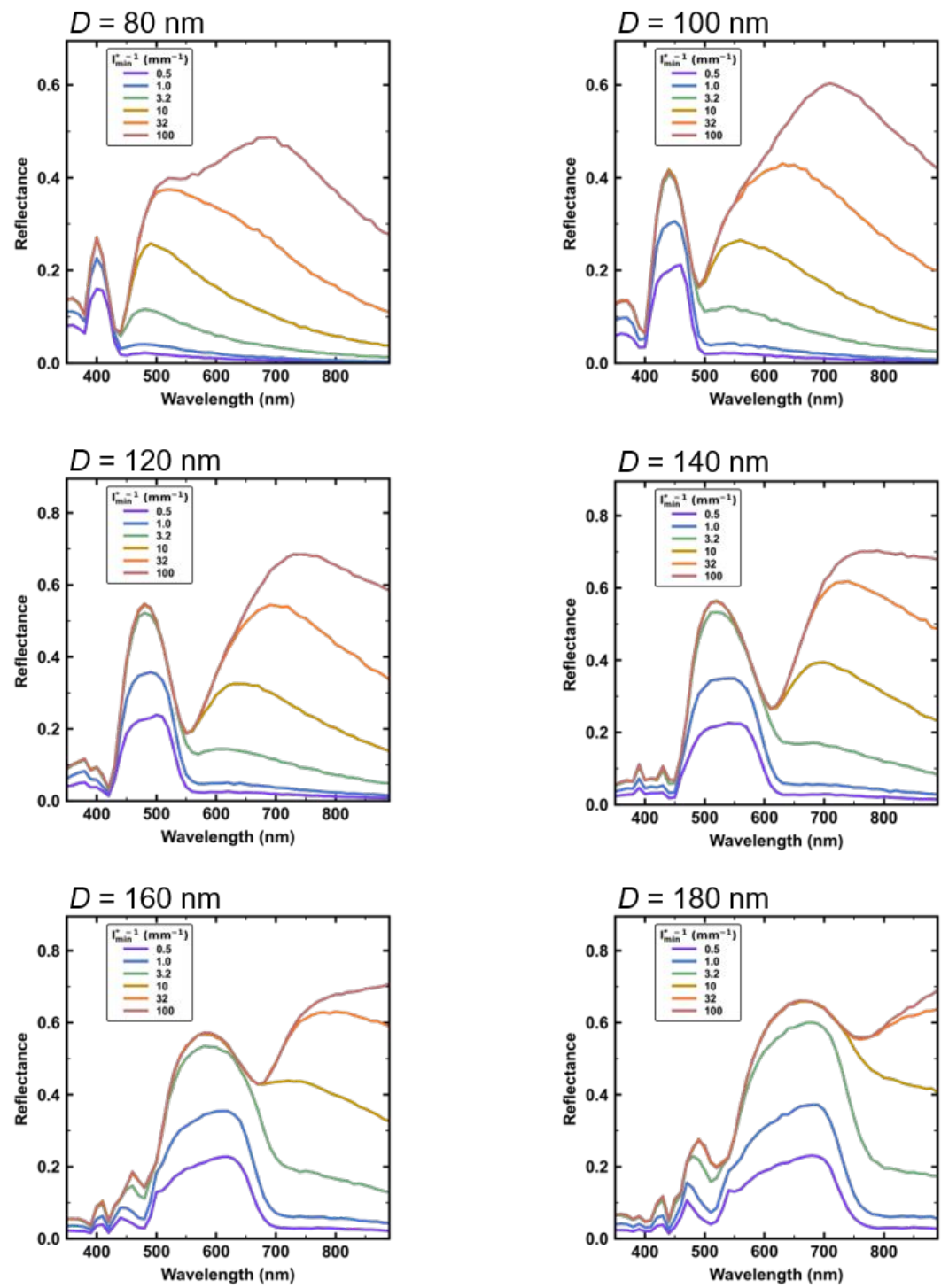

Figure S1. Simulated reflectance spectra of Si NP inks with different diameters and minimum mean free paths. 
(a)

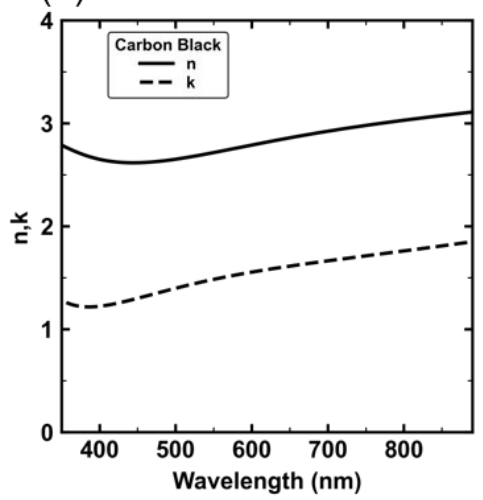

(b)

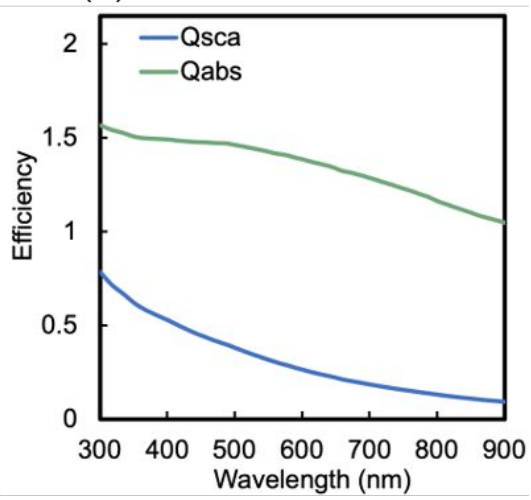

Figure S2. (a) Refractive index and extinction coefficient of amorphous carbon. (b)

Calculated scattering $\left(\mathrm{Q}_{\text {sca }}\right)$ and absorption $\left(\mathrm{Q}_{\mathrm{abs}}\right)$ efficiencies of $110 \mathrm{~nm}$ CB NP.

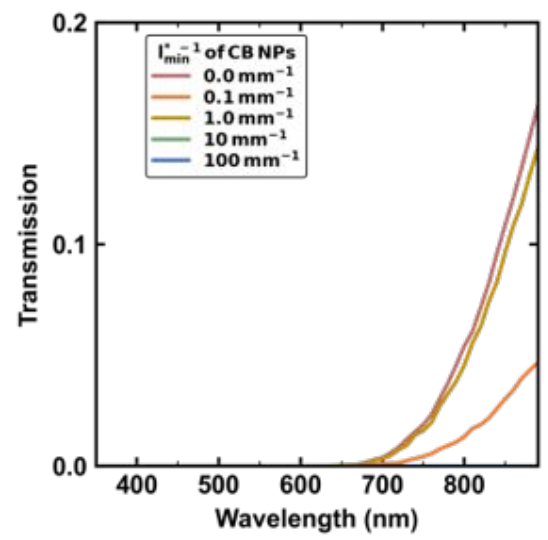

Figure S3. Simulated transmittance spectra of Si NP inks with different CB NP concentrations. 


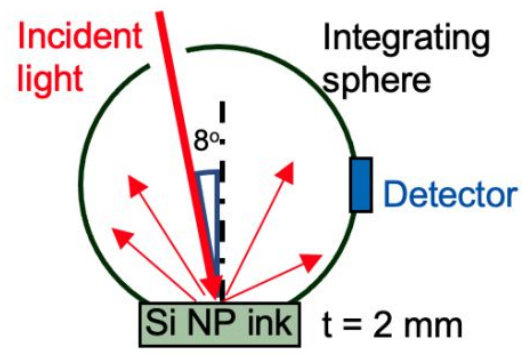

Figure S4. Experimental setup for reflection measurement of Si NP inks.

$117 \mathrm{~nm}$

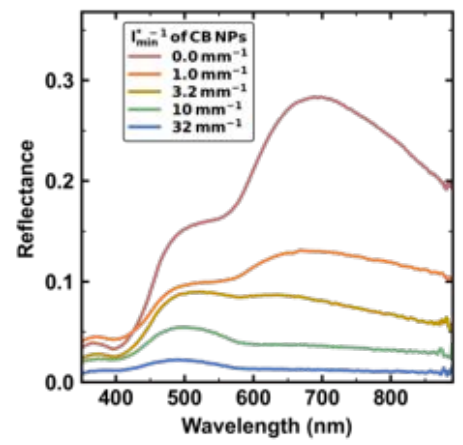

$137 \mathrm{~nm}$

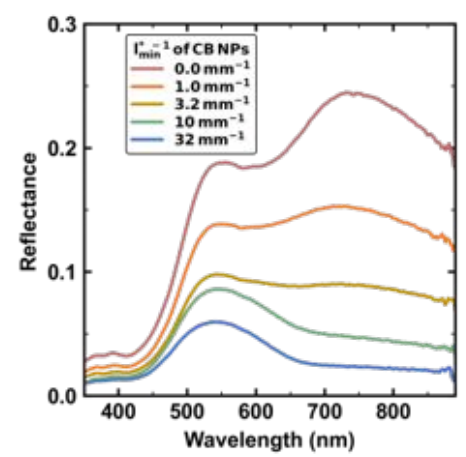

$171 \mathrm{~nm}$

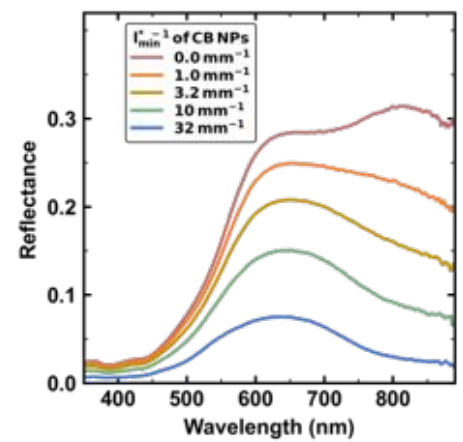

Figure S5. Measured reflection spectra of Si NP inks with different average diameters and CB NP concentrations. 